\title{
Article
}

\section{The Hankel Determinants from a Singularly Perturbed Jacobi Weight}

\author{
Pengju Han ${ }^{1}$ and Yang Chen ${ }^{2, *}$ \\ 1 College of Science, Huazhong Agricultural University, Wuhan 430070, China; hanpengju@mail.hzau.edu.cn \\ 2 Department of Mathematics, Faculty of Science and Technology, University of Macau, Macau 999078, China \\ * Correspondence: yayangchen@um.edu.mo
}

check for updates

Citation: Han, P.; Chen, Y. The Hankel Determinants from a Singularly Perturbed Jacobi Weight. Mathematics 2021, 9, 2978. https:// doi.org/10.3390/math9222978

Academic Editor: Abdelmejid Bayad

Received: 22 September 2021

Accepted: 16 November 2021

Published: 22 November 2021

Publisher's Note: MDPI stays neutral with regard to jurisdictional claims in published maps and institutional affiliations.

Copyright: (C) 2021 by the authors Licensee MDPI, Basel, Switzerland. This article is an open access article distributed under the terms and conditions of the Creative Commons Attribution (CC BY) license (https:// creativecommons.org/licenses/by/ $4.0 /)$.

\begin{abstract}
We study the Hankel determinant generated by a singularly perturbed Jacobi weight $w(x, s):=(1-x)^{\alpha}(1+x)^{\beta} \mathrm{e}^{-\frac{s}{1-x}}, \quad x \in[-1,1], \quad \alpha>0, \quad \beta>0 s \geq 0$. If $s=0$, it is reduced to the classical Jacobi weight. For $s>0$, the factor $\mathrm{e}^{-\frac{s}{1-x}}$ induces an infinitely strong zero at $x=1$. For the finite $n$ case, we obtain four auxiliary quantities $R_{n}(s), r_{n}(s), \widetilde{R}_{n}(s)$, and $\widetilde{r}_{n}(s)$ by using the ladder operator approach. We show that the recurrence coefficients are expressed in terms of the four auxiliary quantities with the aid of the compatibility conditions. Furthermore, we derive a shifted Jimbo-Miwa-Okamoto $\sigma$-function of a particular Painlevé $\mathrm{V}$ for the logarithmic derivative of the Hankel determinant $D_{n}(s)$. By variable substitution and some complicated calculations, we show that the quantity $R_{n}(s)$ satisfies the four Painlevé equations. For the large $n$ case, we show that, under a double scaling, where $n$ tends to $\infty$ and $s$ tends to $0^{+}$, such that $\tau:=n^{2} s$ is finite, the scaled Hankel determinant can be expressed by a particular $P_{I I I^{\prime}}$.
\end{abstract}

Keywords: random matrix theory; Hankel determinant; singularly perturbed Jacobi weight; ladder operators; Painlevé V

\section{Introduction}

Random matrices were introduced in nuclear physics by Wigner in the 1950s to describe the statistics of the energy levels of quantum systems. The theory of random matrices makes the hypothesis that the local statistical behavior of the energy levels is identical to that of the eigenvalues of a random matrix.

In random matrix theory (RMT), it is well known that the joint probability density of the eigenvalues $\left\{x_{j}\right\}_{j=1}^{n}$ of $n \times n$ Hermitian matrices in the unitary ensemble is [1,2]:

$$
p\left(x_{1}, x_{2}, \ldots, x_{n}\right) d x_{1} d x_{2} \cdots d x_{n}=\frac{1}{D_{n}[w]} \frac{1}{n !} \prod_{1 \leq j<k \leq n}\left(x_{j}-x_{k}\right)^{2} \prod_{l=1}^{n} w\left(x_{l}\right) d x_{l}
$$

where $w(x)$ is a weight or probability density supported on the interval $I \subseteq \mathbb{R}$, and we suppose the moments of all orders,

$$
\mu_{j}:=\int_{I} x^{j} w(x) d x, \quad j=0,1,2, \ldots
$$

exist. In addition, $D_{n}[w]$ is the normalization constant or the partition function,

$$
D_{n}[w]=\frac{1}{n !} \int_{I^{n}} \prod_{1 \leq j<k \leq n}\left(x_{j}-x_{k}\right)^{2} \prod_{l=1}^{n} w\left(x_{l}\right) d x_{l} .
$$

As a matter of fact, $D_{n}[w]$ can be expressed as the determinant of the Hankel or moment matrix [3] (2.2.11):

$$
D_{n}[w]=\operatorname{det}\left(\mu_{j+k}\right)_{j, k=0}^{n-1} .
$$


Hankel determinants have been studied extensively over the past few years in part due to connections with RMT [4-13]. This is because Hankel determinants compute the most fundamental objects in RMT, such as the partition function of a random matrix ensemble, the probability distribution function of the largest eigenvalue of Hermitian matrices, and the moment-generating function of linear statistics associated with the ensemble.

The motivation of this paper comes from the study of different single or double compression models in recent years. In an early paper, Chen and Its [14] studied the Laguerre weight $x^{\alpha} \mathrm{e}^{-x}$ perturbing a multiplicative factor $\mathrm{e}^{-s / x}$, which induces an infinitely strong zero at the origin. Then, Chen and Dai considered the Jacobi weight $x^{\alpha}(1-x)^{\beta}$ perturbing a multiplicative factor $\mathrm{e}^{-s / x}$ in the paper [15]. Later, Min and Chen studied two kinds of even weights $\left(1-x^{2}\right)^{\alpha} \mathrm{e}^{-s / x^{2}}$ in [16] and $\left(1-x^{2}\right)^{\alpha} \mathrm{e}^{-s /\left(1-x^{2}\right)}$ in [17]. Zhu and Chen [18] considered a generalized weight function:

$$
w(z)=\left(1-z^{2}\right)^{\alpha} \mathrm{e}^{-t /\left(z^{2}-k^{2}\right)}, z, k \in[-1,1], \alpha, t>0 .
$$

The relationshipbetween semiclassical orthogonal polynomials and the (continuous) Painlevé equations was demonstrated by Magnus [19], who showed that the coefficients in the three-term recurrence relation for the Freud weight [20]:

$$
\omega(x ; t)=\exp \left(-\frac{1}{4} x^{4}-t x^{2}\right), x \in \mathbb{R},
$$

with $t \in \mathbb{R}$ a parameter, can be expressed in terms of solutions of $P_{I V}$. Recently, Wang and Fan [21] studied the large $n$ asymptotics of the monic orthogonal polynomials with respect to another singularly perturbed Pollaczek-Jacobi-type weight:

$$
w(x)=x^{\alpha}(1-x)^{\beta} e^{-\frac{t}{x(x-1)}}, x \in[0,1], \alpha, \beta>0,, t \geq 0 .
$$

In this paper, we consider the Hankel determinant generated by a singularly perturbed Jacobi weight, namely,

$$
D_{n}(s, \alpha, \beta):=\operatorname{det}\left(\int_{-1}^{1} x^{j+k} w(x, s) d x\right)_{j, k=0}^{n-1}
$$

where:

$$
w(x, s):=(1-x)^{\alpha}(1+x)^{\beta} \mathrm{e}^{-\frac{s}{1-x}}, \quad x \in[-1,1], \quad \alpha>0, \quad \beta>0, \quad s \geq 0 .
$$

We call this the single compression model because the weight vanishes infinitely fast at $x=1$.

The study of this Hankel determinant is motivated in part by the Wigner time-delay distribution in chaotic cavities [22]. In recent years, the asymptotic analysis of orthogonal polynomials and Hankel determinants for the singularly perturbed weights has attracted much interest; see, e.g., [10,12,13,21,23,24].

In addition, for the unperturbed Jacobi weight, i.e., $s=0$, we have the explicit formula of $D_{n}(0)$ [2] (17.6.2):

$$
\begin{aligned}
D_{n}(0) & =\frac{2^{n(n+\alpha+\beta)}}{n !} \prod_{j=1}^{n} \frac{\Gamma(j+1) \Gamma(j+\alpha) \Gamma(j+\beta)}{\Gamma(j+n+\alpha+\beta)} \\
& =2^{n(n+\alpha+\beta)} \frac{G(n+1) G(n+1+\alpha+\beta) G(n+\alpha+1) G(n+\beta+1)}{G(2 n+\alpha+\beta+1) G(\alpha+1) G(\beta+1)},
\end{aligned}
$$

where $G(\cdot)$ is the Barnes G-function, which satisfies the relation [25]:

$$
G(z+1)=\Gamma(z) G(z), \Re(z)>0, \quad G(1):=1 .
$$


See also [26] for more properties of this function.

It is well known that $D_{n}(s)$ can be expressed as the product of the square of the $L^{2}$ norms of the monic orthogonal polynomials [27] (2.1.6), namely:

$$
D_{n}(s)=\prod_{j=0}^{n-1} h_{j}(s),
$$

where:

$$
h_{j}(s) \delta_{j k}=\int_{-1}^{1} P_{j}(x, s) P_{k}(x, s) w(x, s) d x, \quad j, k=0,1,2, \ldots
$$

and $P_{j}(x, s), j=0,1,2, \ldots$, are the monic polynomials of degree $j$ orthogonal with respect to the weight function $w(x, s)$.

In Section 2, with the aid of supplementary conditions, we obtain a system of difference equations by certain auxiliary quantities $\left(R_{n}, r_{n}, \widetilde{R}_{n}, \widetilde{r}_{n}\right)$ and the recurrence coefficients $\left(\alpha_{n}, \beta_{n}\right)$. In Section 3.1, we make use of the results of Section 2 to express $\alpha_{n}$ and $\beta_{n}$ in terms of the auxiliary quantities and show that these reduce to recurrence coefficients of the classical Jacobi weight when $s=0$. A pair of Toda equations is derived in Section 3.2 by the $s$ dependence of the recurrence coefficients and the auxiliary quantities. In Section 4, we express $\widetilde{r}_{n}, r_{n}$ and $R_{n}$ in terms of:

$$
H_{n}(s):=s \frac{d}{d s} \ln D_{n}(s)
$$

and $H_{n}^{\prime}(s)$. We show that:

$$
\widetilde{H}_{n}(t):=\left.H_{n}(s)\right|_{s=2 t}-n(n+\alpha+\beta)
$$

satisfies the Jimbo-Miwa-Okamoto $\sigma$-form of a Painlevé V equation. In Section 5, we derive a Painlevé V form by $S_{n}(t):=\left.2 R_{n}(s)\right|_{s=2 t} /(2 n+\alpha+\beta+1)$. In Section 6, we use the double-scaling analysis and obtain some new results. Finally, Section 7 ends this paper with a brief conclusion.

\section{Preliminaries}

The main method of this paper is the ladder operator approach associated with the orthogonal polynomials. This approach has been widely applied to solve the problems on Hankel determinants generated by various weight functions; see $[10,14,15,28,29]$ for reference. Therefore, we introduce some elementary facts about the orthogonal polynomials.

Let $P_{n}(x)$ be the monic polynomials of degree $n$ in $x$ and orthogonal with respect to the weight function $w(x, s)$ defined in (1), that is:

$$
\int_{-1}^{1} P_{m}(x) P_{n}(x) w(x, s) d x=h_{n} \delta_{m, n} .
$$

Here, the polynomials $P_{n}(x)$ and the constant $h_{n}$ all depend on $s$, but we suppress the dependence for brevity. An immediate consequence of the orthogonality condition is the recurrence relation [3]:

$$
x P_{n}(x)=P_{n+1}(x)+\alpha_{n} P_{n}(x)+\beta_{n} P_{n-1}(x), \quad n=0,1, \ldots
$$

with the initial conditions $P_{0}(z):=1$ and $\beta_{0} P_{-1}(z):=0$. The most commonly used orthogonal polynomials are orthogonal for a measure with support in a real interval. The classical orthogonal polynomials include Jacobi polynomials, Laguerre polynomials, Hermite polynomials, and their special cases, Gegenbauer polynomials, Chebyshev polynomials, and Legendre polynomials. Note that $P_{n}(z)$ has the following form [30]:

$$
P_{n}(z)=z^{n}+\mathrm{p}_{1}(n) z^{n-1}+\ldots
$$


Substituting (7) into (6), we see that:

$$
\alpha_{n}=\mathrm{p}_{1}(n)-\mathrm{p}_{1}(n+1) .
$$

Taking a telescopic sum of the above equation and noting that $\mathrm{p}_{1}(0):=0$, we have:

$$
-\sum_{j=0}^{n-1} \alpha_{j}=\mathrm{p}_{1}(n)
$$

The Hankel determinant generated by our weight is:

$$
\begin{aligned}
D_{n}(s): & =\operatorname{det}\left(\mu_{j+k}(s)\right)_{j, k=0}^{n-1} \\
& =\prod_{j=0}^{n-1} h_{j},
\end{aligned}
$$

where:

$$
\mu_{k}(s):=\int_{-1}^{1} x^{k}(1-x)^{\alpha}(1+x)^{\beta} \mathrm{e}^{-\frac{s}{1-x}} d x .
$$

For the case $k=0$, we have:

$$
\mu_{0}(s)=2^{\alpha+\beta+1} \mathrm{e}^{-\frac{s}{2}} \Gamma(1+\beta) U\left(1+\beta,-\alpha, \frac{s}{2}\right)
$$

and $U(a, b, z)$ is the Kummer function of the second; see [26,31].

The Hankel determinant will turn out to play an important role in our determination of $\alpha_{n}$ and $\beta_{n}$ for the weight given by (1). Furthermore, through several auxiliary variables, we obtain expressions for the $\alpha_{n}$ and $\beta_{n}$ terms of $H_{n}(s)$ given in (1.2) and its derivatives with respect to $s$. Equations (5)-(10) can be found in Szegö's treatise [3] on orthogonal polynomials.

In this section, we introduce a pair of ladder operators and the compatibility conditions first. The information below can be found in Chen and Its [14].

Suppose that $w(x)$ is a continuous even weight function defined on $[-1,1]$, and $w(-1)=w(1)=0$. The monic orthogonal polynomials with respect to $w(x)$ satisfy the lowering operator equation:

$$
\left(\frac{d}{d z}+B_{n}(z)\right) P_{n}(z)=\beta_{n} A_{n}(z) P_{n-1}(z)
$$

and the raising operator equation:

$$
\left(\frac{d}{d z}-B_{n}(z)-\mathrm{v}^{\prime}(z)\right) P_{n-1}(z)=-A_{n-1}(z) P_{n}(z),
$$

where $\mathrm{v}(z):=-\ln w(z)$, and:

$$
\begin{gathered}
A_{n}(z):=\frac{1}{h_{n}} \int_{-1}^{1} \frac{\mathrm{v}^{\prime}(z)-\mathrm{v}^{\prime}(y)}{z-y} P_{n}^{2}(y) w(y) d y, \\
B_{n}(z):=\frac{1}{h_{n-1}} \int_{-1}^{1} \frac{\mathrm{v}^{\prime}(z)-\mathrm{v}^{\prime}(y)}{z-y} P_{n}(y) P_{n-1}(y) w(y) d y .
\end{gathered}
$$

The functions $A_{n}(z)$ and $B_{n}(z)$ are not independent, but must satisfy the following supplementary conditions valid for $z \in \mathbb{C} \cup\{\infty\}$ :

$$
B_{n+1}(z)+B_{n}(z)=\left(z-\alpha_{n}\right) A_{n}(z)-\mathrm{v}^{\prime}(z),
$$




$$
\begin{gathered}
1+\left(z-\alpha_{n}\right)\left(B_{n+1}(z)-B_{n}(z)\right)=\beta_{n+1} A_{n+1}(z)-\beta_{n} A_{n-1}(z), \\
B_{n}^{2}(z)+\mathrm{v}^{\prime}(z) B_{n}(z)+\sum_{j=0}^{n-1} A_{j}(z)=\beta_{n} A_{n}(z) A_{n-1}(z) .
\end{gathered}
$$

In our problem, the weight and associated quantities are:

$$
\begin{gathered}
w(x, s)=(1-x)^{\alpha}(1+x)^{\beta} \mathrm{e}^{-\frac{s}{1-x}}, \\
\mathrm{v}(z)=-\ln w(x, s)=\frac{s}{1-z}-\alpha \ln (1-z)-\beta \ln (1+z), \quad \mathrm{v}^{\prime}(z)=\frac{\alpha}{1-z}-\frac{\beta}{1+z}+\frac{s}{(1-z)^{2}}, \\
\frac{\mathrm{v}^{\prime}(z)-\mathrm{v}^{\prime}(y)}{z-y}=\frac{1}{1-z}\left(\frac{\alpha}{1-y}+\frac{s}{(1-y)^{2}}\right)+\frac{\beta}{(1+z)(1+y)}+\frac{s}{(1-z)^{2}(1-y)} .
\end{gathered}
$$

Substituting the above formula into the definition of $A_{n}(z)$ and $B_{n}(z)$ in (13) and (14), we have the following proposition. In the following discussions, we suppress the $s$ dependence for brevity.

Proposition 1. For our problem, we have:

$$
\begin{aligned}
& A_{n}(z)=\frac{R_{n}}{1-z}+\frac{R_{n}}{1+z}+\frac{\widetilde{R}_{n}}{(1-z)^{2}}, \\
& B_{n}(z)=\frac{r_{n}+n}{1-z}+\frac{r_{n}}{1+z}+\frac{\widetilde{r}_{n}}{(1-z)^{2}},
\end{aligned}
$$

where:

$$
\begin{gathered}
\widetilde{R}_{n}(s):=\frac{s}{h_{n}} \int_{-1}^{1} \frac{P_{n}^{2}(y) w(y)}{1-y} d y, \\
R_{n}(s):=\frac{\beta}{h_{n}} \int_{-1}^{1} \frac{P_{n}^{2}(y) w(y)}{1+y} d y, \\
\widetilde{r}_{n}(s):=\frac{s}{h_{n-1}} \int_{-1}^{1} \frac{P_{n}(y) P_{n-1}(y) w(y)}{1-y} d y, \\
r_{n}(s):=\frac{\beta}{h_{n-1}} \int_{-1}^{1} \frac{P_{n}(y) P_{n-1}(y) w(y)}{1+y} d y,
\end{gathered}
$$

Proof. According to the definition of $A_{n}(z)$ in (13), we obtain:

$$
\begin{aligned}
A_{n}(z) & =\frac{1}{1-z} \frac{1}{h_{n}} \int_{-1}^{1} P_{n}^{2}(y) w(y)\left(\frac{\alpha}{1-y}+\frac{s}{(1-y)^{2}}\right) d y+\frac{1}{1+z} \frac{\beta}{h_{n}} \int_{-1}^{1} P_{n}^{2}(y) w(y) \frac{d y}{1+y} \\
& +\frac{s}{(1-z)^{2}} \frac{1}{h_{n}} \int_{-1}^{1} P_{n}^{2}(y) w(y) \frac{d y}{1-y} .
\end{aligned}
$$

Applying integration by parts, we have:

$$
\int_{-1}^{1} P_{n}^{2}(y) w(y) \frac{\alpha}{1-y} d y=\beta \int_{-1}^{1} P_{n}^{2}(y) w(y) \frac{d y}{1+y}-\int_{-1}^{1} P_{n}^{2}(y) w(y) \frac{s}{(1-y)^{2}} d y
$$

Combining (27) and (28) gives us (21).

Similarly, we obtain (22) from (14). One just needs to take into account the following equality: 


$$
\begin{aligned}
& \int_{-1}^{1} P_{n}(y) P_{n-1}(y) w(y) \frac{\alpha}{1-y} d y \\
& =n h_{n-1}+\int_{-1}^{1} P_{n}(y) P_{n-1}(y) w(y) \frac{\beta}{1+y} d y-\int_{-1}^{1} P_{n}(y) P_{n-1}(y) w(y) \frac{s}{(1-y)^{2}} d y
\end{aligned}
$$

This completes the proof.

Now, we have four more auxiliary quantities $R_{n}, \widetilde{R}_{n}, r_{n}, \widetilde{r}_{n}$, in addition to the two unknowns $\alpha_{n}$ and $\beta_{n}$. However, from (15)-(17), we obtain relations among these quantities.

Proposition 2. From (15), we obtain the following equations:

$$
\begin{aligned}
& \widetilde{r}_{n+1}+\widetilde{r}_{n}=\left(1-\alpha_{n}\right) \widetilde{R}_{n}-s, \\
& \widetilde{R}_{n}=2 R_{n}-\alpha-\beta-2 n-1, \\
& r_{n+1}+r_{n}=\beta-\left(1+\alpha_{n}\right) R_{n},
\end{aligned}
$$

where the constants $R_{n}, \widetilde{R}_{n}, r_{n}$, and $\widetilde{r}_{n}$ are defined in (23)-(26), respectively.

Proof. Substituting (21) and (22) into (15), we obtain:

$$
B_{n+1}(z)+B_{n}(z)=\frac{r_{n+1}+r_{n}+2 n+1}{1-z}+\frac{r_{n+1}+r_{n}}{1+z}+\frac{\widetilde{r}_{n+1}+\widetilde{r}_{n}}{(1-z)^{2}}
$$

and:

$$
\begin{aligned}
& \left(z-\alpha_{n}\right) A_{n}(z)-\mathrm{v}^{\prime}(z)=\left(z-\alpha_{n}\right)\left[\frac{R_{n}}{1-z}+\frac{R_{n}}{1+z}+\frac{\widetilde{R}_{n}}{(1-z)^{2}}\right]-\left[\frac{\alpha}{1-z}-\frac{\beta}{1+z}+\frac{s}{(1-z)^{2}}\right] \\
& =-R_{n}+\frac{\left(1-\alpha_{n}\right) R_{n}-\alpha-\widetilde{R}_{n}}{1-z}+\frac{\left(z-\alpha_{n}\right) R_{n}+\beta}{1+z}+\frac{\left(1-\alpha_{n}\right) \widetilde{R}_{n}-s}{(1-z)^{2}} .
\end{aligned}
$$

Comparing the coefficients in the above two formulas, it follows that:

$$
\begin{gathered}
\widetilde{r}_{n+1}+\widetilde{r}_{n}=\left(1-\alpha_{n}\right) \widetilde{R}_{n}-s, \\
r_{n+1}+r_{n}+2 n+1=-\alpha+\left(1-\alpha_{n}\right) R_{n}-\widetilde{R}_{n}, \\
r_{n+1}+r_{n}=\beta-\left(1+\alpha_{n}\right) R_{n} .
\end{gathered}
$$

Combining the above three formulas immediately proves our proposition.

Proposition 3. From (17), we obtain the following equations:

$$
\begin{aligned}
\left(\widetilde{r}_{n}\right)^{2}+s \widetilde{r}_{n} & =\beta_{n} \widetilde{R}_{n} \widetilde{R}_{n-1} \\
\left(2 \widetilde{r}_{n}+s\right)\left(r_{n}+n\right)+\alpha \widetilde{r}_{n} & =\beta_{n}\left(R_{n} \widetilde{R}_{n-1}+R_{n-1} \widetilde{R}_{n}\right), \\
r_{n}^{2}-\beta r_{n} & =\beta_{n} R_{n} R_{n-1}
\end{aligned}
$$

and:

$$
2 \sum_{j=0}^{n-1} \widetilde{R}_{j}=(2 n+\alpha+\beta)\left(\widetilde{r}_{n}-2 r_{n}\right)+n(s-2 \alpha-2 n)
$$

where the constants $R_{n}, \widetilde{R}_{n}, r_{n}$, and $\widetilde{r}_{n}$ are defined in (23)-(26), respectively. 
Proof. From (21) and (22), we know that:

$$
\begin{aligned}
& B_{n}^{2}(z)+\mathrm{v}^{\prime}(z) B_{n}(z)+\sum_{j=0}^{n-1} A_{j}(z) \\
= & \frac{\widetilde{r}_{n}^{2}+s \widetilde{r}_{n}}{(1-z)^{4}}+\frac{\left(2 \widetilde{r}_{n}+s\right)\left(r_{n}+n\right)+\alpha \widetilde{r}_{n}}{(1-z)^{3}}+\frac{\left(r_{n}+n\right)\left(r_{n}+n+\alpha\right)}{(1-z)^{2}} \\
+ & \frac{r_{n}^{2}-\beta r_{n}}{(1+z)^{2}}+\frac{2 \widetilde{r}_{n} r_{n}-\beta \widetilde{r}_{n}+s r_{n}}{(1+z)(1-z)^{2}}+\frac{\left(r_{n}+n\right)\left(2 r_{n}-\beta\right)+\alpha r_{n}}{(1-z)(1+z)} \\
+ & \sum_{j=0}^{n-1}\left[\frac{R_{j}}{1-z}+\frac{R_{j}}{1+z}+\frac{\widetilde{R}_{j}}{(1-z)^{2}}\right] .
\end{aligned}
$$

Using (21) again, we obtain:

$$
\begin{aligned}
\beta_{n} A_{n}(z) A_{n-1}(z) & =\frac{\beta_{n} \widetilde{R}_{n} \widetilde{R}_{n-1}}{(1-z)^{4}}+\beta_{n} R_{n} R_{n-1}\left[\frac{1}{(1-z)^{2}}+\frac{1}{(1+z)^{2}}+\frac{2}{(1-z)(1+z)}\right] \\
& +\beta_{n}\left(R_{n} \widetilde{R}_{n-1}+\widetilde{R}_{n} R_{n-1}\right)\left[\frac{1}{(1-z)^{3}}+\frac{1}{(1+z)(1-z)^{2}}\right] .
\end{aligned}
$$

Note that (42) equals (43) due to (17). Then, let us compare their coefficients. At $\mathcal{O}(1-z)^{-4}, \mathcal{O}(1-z)^{-3}$, and $\mathcal{O}(1+z)^{-2}$, equating the coefficients, we have (38)-(40), respectively. At $\mathcal{O}(1-z)^{-2}$, we obtain:

$$
\begin{aligned}
& 2\left(r_{n}+n\right)\left(r_{n}+n+\alpha\right)+2 \widetilde{r}_{n} r_{n}-\beta \widetilde{r}_{n}+s r_{n}+2 \sum_{j=0}^{n-1} \widetilde{R}_{j} \\
= & \beta_{n}\left(R_{n} \widetilde{R}_{n-1}+\widetilde{R}_{n} R_{n-1}\right)+2 \beta_{n} R_{n} R_{n-1} .
\end{aligned}
$$

Substituting (39) and (40) into (44) gives us (41).

From (16), using calculations similar to those in the above proposition, we obtain one more equation as follows:

$$
2\left(r_{n+1}-r_{n}\right)-\left(\widetilde{r}_{n+1}-\widetilde{r}_{n}\right)+1-\alpha_{n}=0 .
$$

To continue, we rewrite (45) as:

$$
\alpha_{n}=2\left(r_{n+1}-r_{n}\right)-\left(\widetilde{r}_{n+1}-\widetilde{r}_{n}\right)+1 .
$$

Performing a telescopic sum and recalling (9), we find the very handy relation:

$$
\mathrm{p}_{1}(n)=\widetilde{r}_{n}-2 r_{n}-n,
$$

where we used the initial conditions $r_{0}(t)=\widetilde{r}_{0}(t):=0$. As we shall see later, (47) will play a crucial role in the derivation of the Painlevé equation.

\section{Ladder Operator Approach}

\subsection{The Recurrence Coefficients}

In this section, we express the recurrence coefficients $\alpha_{n}$ and $\beta_{n}$ in terms of the auxiliary quantities $R_{n}, r_{n}$, and $\widetilde{r}_{n}$. 
Lemma 1. The diagonal recurrence coefficients $\alpha_{n}$ are expressed in terms of $R_{n}, r_{n}$, and $\widetilde{r}_{n}$ as follows:

$$
(2 n+\alpha+\beta+2) \alpha_{n}=2\left(\widetilde{r}_{n}-2 r_{n}\right)-4 R_{n}+\alpha+3 \beta+2 n+s+2 .
$$

Proof. We eliminate $\widetilde{R}_{n}$ from (30) with the aid of (31) and find:

$$
\widetilde{r}_{n}+\widetilde{r}_{n+1}=\left(1-\alpha_{n}\right)\left(2 R_{n}-\alpha-\beta-2 n-1\right)-s .
$$

Subtracting the above formula from (32), we obtain:

$$
\widetilde{r}_{n}+\widetilde{r}_{n+1}-2\left(r_{n+1}+r_{n}\right)=\left(1-\alpha_{n}\right)(-\alpha-\beta-2 n-1)-s-2 \beta+4 R_{n} .
$$

Recalling (45), we see that the left-hand side of the above formula is $2\left(\widetilde{r}_{n}-2 r_{n}\right)+1-$ $\alpha_{n}$. Then, (48) immediately follows.

Remark 1. For $n=0$, we find from the definition of $\alpha_{0}(s)$ and $R_{0}(s)$ that:

$$
\begin{aligned}
& \alpha_{0}(s)=1-\frac{2 U\left(1+\beta,-\alpha-1, \frac{s}{2}\right)}{U\left(1+\beta,-\alpha, \frac{s}{2}\right)}, \\
& R_{0}(s)=\frac{U\left(\beta,-\alpha, \frac{s}{2}\right)}{2 U\left(1+\beta,-\alpha, \frac{s}{2}\right)},
\end{aligned}
$$

where $U$ is the second solution of Kummer's equation; see [26]. We verify the validity of (48) at $n=0$ by substituting the above two formulas.

The next lemma gives an expression for $\beta_{n}$.

Lemma 2. The off-diagonal recurrence coefficients $\beta_{n}$ are expressed in terms of $r_{n}$ and $\widetilde{r}_{n}$ as follows:

$$
\left[(2 n+\alpha+\beta)^{2}-1\right] \beta_{n}=\left(\widetilde{r}_{n}-2 r_{n}\right)^{2}+(s-2 \alpha-4 n) \widetilde{r}_{n}-2(s+2 \beta) r_{n}-2 n s .
$$

Proof. We eliminate $\widetilde{R}_{n}$ from (38) with the aid of (31) and replace $\beta_{n} R_{n} R_{n-1}$ by $r_{n}^{2}-\beta r_{n}$ with (39) to find:

$$
\begin{aligned}
& \left(\widetilde{r}_{n}\right)^{2}+s \widetilde{r}_{n}=\beta_{n}\left(2 R_{n}-(\alpha+\beta+2 n+1)\right)\left(2 R_{n-1}-(\alpha+\beta+2 n-1)\right) \\
= & 4 \beta_{n} R_{n} R_{n-1}-2 \beta_{n}\left[(\alpha+\beta+2 n+1) R_{n-1}+(\alpha+\beta+2 n-1) R_{n}\right]+\left[(2 n+\alpha+\beta)^{2}-1\right] \beta_{n} .
\end{aligned}
$$

The same substitutions as that made in (40) produce:

$$
\begin{aligned}
\left(r_{n}+n\right)\left(2 \widetilde{r}_{n}+s\right)+\alpha \widetilde{r}_{n} & =\beta_{n}\left[R_{n}\left(2 R_{n-1}-(\alpha+\beta+2 n-1)\right)+R_{n-1}\left(2 R_{n}-(\alpha+\beta+2 n+1)\right)\right] \\
& =4 \beta_{n} R_{n} R_{n-1}-\beta_{n}\left[(\alpha+\beta+2 n+1) R_{n-1}+(\alpha+\beta+2 n-1) R_{n}\right] .
\end{aligned}
$$

Subtracting the above two formulas gives us (53).

Remark 2. We consider the case when $s=0$. In this situation, $\widetilde{R}_{n}(0)=\widetilde{r}_{n}(0)=0$. Therefore, from (31) and (41), we find:

$$
R_{n}(0)=\frac{\alpha+\beta+2 n+1}{2}
$$

and:

$$
r_{n}(0)=\frac{-n(n+\alpha)}{2 n+\alpha+\beta}
$$


respectively. Finally, from (48) and (53), we obtain:

$$
\begin{gathered}
\alpha_{n}(0)=\frac{\beta^{2}-\alpha^{2}}{(2 n+\alpha+\beta+2)(2 n+\alpha+\beta)}, \\
\beta_{n}(0)=\frac{4 n(n+\alpha)(n+\beta)(n+\alpha+\beta)}{(\alpha+\beta+2 n)^{2}\left[(2 n+\alpha+\beta)^{2}-1\right]} .
\end{gathered}
$$

They are in agreement with the classical theory in [32].

\subsection{The s Dependence}

Note that our weight function depends on $s$. As a consequence, the coefficients of our polynomials, the recurrence coefficients, and the auxiliary quantities defined in (23)-(26) all depend on $s$. In this section, we study the evolution of auxiliary quantities in $s$. First of all, we state a lemma that concerns the derivative of $\mathrm{p}_{1}(n)$ with respect to $s$.

Lemma 3. We have:

$$
s \frac{d}{d s} \mathrm{p}_{1}(n)=\widetilde{r}_{n} .
$$

Proof. By the orthogonal property (5), we know that:

$$
\int_{-1}^{1} P_{n}(x) P_{n-1}(x) w(x, s) d x=0 .
$$

Differentiating the above formula with respect to $s$ gives:

$$
\int_{-1}^{1} \frac{d}{d s} P_{n}(x) P_{n-1}(x) w(x, s) d x-\int_{-1}^{1} P_{n}(x) P_{n-1}(x) \frac{d}{d s} w(x, s) d x=0 .
$$

Using (1), (5), and (7), we obtain:

$$
h_{n-1} \frac{d}{d s} \mathrm{p}_{1}(n)-\int_{-1}^{1} P_{n}(x) P_{n-1}(x) w(x, s) \frac{1}{1-x} d x=0 .
$$

Taking into account (25), we obtain (59) immediately.

From (47) and the above lemma, it is easily seen that:

$$
s \frac{d}{d s} \mathrm{p}_{1}(n)=\widetilde{r}_{n}=s \frac{d}{d s} \widetilde{r}_{n}-2 s \frac{d}{d s} r_{n}
$$

or:

$$
s \frac{d}{d s} \widetilde{r}_{n}=\widetilde{r}_{n}+2 s \frac{d}{d s} r_{n} .
$$

We introduce a quantity related to the logarithmic derivative of the Hankel determinant,

$$
H_{n}(s):=s \frac{d}{d s} \ln D_{n}(s) .
$$

Next, we have the following property concerning the Hankel determinant $D_{n}$.

Lemma 4. We have:

$$
H_{n}(s)=s \frac{d}{d s} \ln D_{n}(s)=-\sum_{j=0}^{n-1} \widetilde{R}_{j}
$$

where $\widetilde{R}_{j}$ is defined in (23). 
Proof. Note that the constant $h_{n}$ defined in (5) depends on the parameter $s$. Then, we have from (1) and (5):

$$
h_{n}^{\prime}(s)=-\int_{-1}^{1} P_{n}^{2}(x) w(x, s) \frac{d x}{1-x} .
$$

Using (23), we obtain from the above formula:

$$
h_{n}^{\prime}=-\frac{\widetilde{R}_{n} h_{n}}{s},
$$

which gives us:

$$
s \frac{d}{d s} \ln h_{n}=-\widetilde{R}_{n} .
$$

Then, our lemma follows from the above formula and (10).

From the above lemmas, we also derive differential relations for the recurrence coefficients $\alpha_{n}$ and $\beta_{n}$. These are the nonstandard Toda equations.

Lemma 5. We have:

$$
\begin{gathered}
s \frac{d}{d s} \alpha_{n}=\widetilde{r}_{n}-\widetilde{r}_{n+1}, \\
s \frac{d}{d s} \beta_{n}=\left(\widetilde{R}_{n-1}-\widetilde{R}_{n}\right) \beta_{n},
\end{gathered}
$$

where $\widetilde{R}_{n}$ and $\widetilde{r}_{n}$ are defined in (23) and (25), respectively.

Proof. ( $\left.T_{1}\right)$ follows from (8) and (59). ( $\left.T_{2}\right)$ follows from (65) and the fact that $\beta_{n}=$ $h_{n} / h_{n-1}$.

\section{The Jimbo-Miwa-Okamoto $\sigma$-form of the Painlevé V}

In this section, we study the logarithmic derivative of the Hankel determinant. Usually, this quantity satisfies a second-order differential equation, which is related to the JimboMiwa-Okamoto $\sigma$-form of a particular Painlevé equation. For this purpose, we express $\widetilde{r}_{n}, r_{n}$, and $R_{n}$ in terms of $H_{n}$ and its derivative with respect to $s$ and obtain a functional equation involving $H_{n}, H_{n}^{\prime}$, and $H_{n}^{\prime \prime}$. Firstly, we express $r_{n}$ and $\widetilde{r}_{n}$ in terms of $H_{n}$ and $H_{n}^{\prime}$ in the next lemma.

Lemma 6.

$$
\begin{gathered}
\widetilde{r}_{n}=\frac{-s\left(2 H_{n}^{\prime}+n\right)}{2 n+\alpha+\beta}, \\
r_{n}=\frac{H_{n}-s H_{n}^{\prime}-n(n+\alpha)}{2 n+\alpha+\beta} .
\end{gathered}
$$

Proof. From (41) and (63), we obtain:

$$
\begin{aligned}
-2 H_{n} & =n s-2(\alpha+n) n+(2 n+\alpha+\beta)\left(\widetilde{r}_{n}-2 r_{n}\right) \\
& =n s-2(\alpha+n) n+(2 n+\alpha+\beta)\left(n+\mathrm{p}_{1}(n)\right) .
\end{aligned}
$$

Taking the derivative of the above formula with respect to $s$ and using (59), we have:

$$
-2 H_{n}^{\prime}=n+(2 n+\alpha+\beta) \frac{\widetilde{r}_{n}}{s} .
$$

Equation (67) then follows from the above one. Equation (68) follows from eliminating $\widetilde{r}_{n}$ from (69) and (70). 
Remark 3. The logarithmic derivative of the Hankel determinant $H_{n}(s)$ plays an important role. The auxiliary quantities $\widetilde{r}_{n}$ and $r_{n}$ can be expressed in terms of $H_{n}$ and its derivative.

Then, we try to obtain a similar lemma for $R_{n}$. To achieve this, we first derive the relation among $R_{n}, r_{n}$, and $\widetilde{r}_{n}$.

\section{Proposition 4.}

$\frac{\alpha+\beta+2 n+1}{R_{n}}\left(r_{n}^{2}-\beta r_{n}\right)+(\alpha+\beta+2 n-1) \beta_{n} R_{n}=4\left(r_{n}^{2}-\beta r_{n}\right)-\alpha \widetilde{r}_{n}-\left(r_{n}+n\right)\left(2 \widetilde{r}_{n}+s\right)$

and:

$$
\begin{aligned}
\frac{\Delta\left(r_{n}^{2}-\beta r_{n}\right)}{R_{n}}-\Delta \beta_{n} R_{n} & =-4\left(r_{n}^{2}-\beta r_{n}\right)+\alpha \widetilde{r}_{n}+\left(r_{n}+n\right)\left(2 \widetilde{r}_{n}+s\right)-2 s(\alpha+\beta+2 n) \frac{d r_{n}}{d s} \\
\text { where } \Delta: & :(2 n+\alpha+\beta)^{2}-1 .
\end{aligned}
$$

Proof. Substituting (39) into (54) gives us (71). Then, we look back to $\left(T_{2}\right)$ by using (31) and (39):

$$
\begin{aligned}
s \frac{d}{d s} \beta_{n} & =\left(\widetilde{R}_{n-1}-\widetilde{R}_{n}\right) \beta_{n} \\
& =\left(2 R_{n-1}-2 R_{n}+2\right) \beta_{n} \\
& =2 \beta_{n}-2 R_{n} \beta_{n}+2 \frac{r_{n}^{2}-\beta r_{n}}{R_{n}} .
\end{aligned}
$$

Applying $s \frac{d}{d s}$ to (53) gives us:

$$
\begin{aligned}
& (2 n+\alpha+\beta+1)(2 n+\alpha+\beta-1) s \frac{d}{d s} \beta_{n} \\
= & \left(2 \widetilde{r}_{n}+s\right)\left(\widetilde{r}_{n}-2 r_{n}\right)-2 s n+(s-2 \alpha-4 n) \widetilde{r}_{n}-4 s(\alpha+\beta+2 n) \frac{d r_{n}}{d s},
\end{aligned}
$$

where we made use of (60) to arrive at the last step. Substituting (73) into the above formula and eliminating $\beta_{n}$ with (53) gives (72).

From the above proposition, we express $R_{n}$ and $1 / R_{n}$ in terms of $r_{n}, \widetilde{r}_{n}$, and $r_{n}^{\prime}(s)$.

\section{Proposition 5.}

$$
\begin{gathered}
R_{n}=\frac{(2 n+\alpha+\beta+1)\left[4\left(r_{n}^{2}-\beta r_{n}\right)-\alpha \widetilde{r}_{n}-\left(r_{n}+n\right)\left(2 \widetilde{r}_{n}+s\right)+2 s r_{n}^{\prime}(s)\right]}{2\left[\left(\widetilde{r}_{n}-2 r_{n}\right)^{2}+(s-2 \alpha-4 n) \widetilde{r}_{n}-2(s+2 \beta) r_{n}-2 s n\right]}, \\
\frac{1}{R_{n}}=\frac{4\left(r_{n}^{2}-\beta r_{n}\right)-\alpha \widetilde{r}_{n}-\left(r_{n}+n\right)\left(2 \widetilde{r}_{n}+s\right)-2 s r_{n}^{\prime}(s)}{2(\alpha+\beta+2 n+1)\left(r_{n}^{2}-\beta r_{n}\right)} .
\end{gathered}
$$

Proof. These are found by solving for $R_{n}$ and $1 / R_{n}$ from (71) and (72).

Finally, we arrive at the following theorem.

Theorem 1. The logarithmic derivative of the Hankel determinant with respect to s,

$$
H_{n}(s):=s \frac{d}{d s} \ln D_{n}(s)
$$


satisfies a shifted Jimbo-Miwa-Okamoto $\sigma$-form of the Painlevé equation:

$$
\begin{aligned}
& 4\left(s H_{n}^{\prime \prime}\right)^{2}=-16 s\left(H_{n}^{\prime}\right)^{3}+\left(H_{n}^{\prime}\right)^{2}\left[16 H_{n}+(s+2 \alpha)^{2}+8 \beta s\right] \\
& +2 H_{n}^{\prime}\left[(-s-2 \alpha-4 \beta) H_{n}+(s+2 \alpha) n(n+\alpha+\beta)\right]+\left[H_{n}-n(n+\alpha+\beta)\right]^{2},
\end{aligned}
$$

with the boundary conditions:

$$
H_{n}(0)=0, \quad H_{n}^{\prime}(0)=\frac{-n(n+\alpha+\beta)}{2 \alpha} .
$$

Proof. Multiplying (75) and (76) gives us:

$$
\begin{aligned}
4 s^{2}\left(r_{n}^{\prime}\right)^{2}= & s^{2} r_{n}^{2}+r_{n}\left[2 n s^{2}+2 s(\alpha+2 \beta+4 n) \widetilde{r}_{n}\right. \\
& \left.+4(\alpha+\beta+2 n) \widetilde{r}_{n}^{2}\right]+\left[(\alpha+2 n) \widetilde{r}_{n}+n s\right]^{2} .
\end{aligned}
$$

Substituting (67) and (68) into (78) gives us (77).

Remark 4. It turns out that the quantity $H_{n}(s)$ satisfies a shifted Jimbo-Miwa-Okamoto $\sigma$-form of the Painlevé $V$ for a special choice for parameters. If:

$$
\tilde{H}_{n}(t):=H_{n}(2 t)-n(n+\alpha+\beta),
$$

then (77) becomes:

$$
\begin{aligned}
\left(t \tilde{H}_{n}^{\prime \prime}\right)^{2} & =-4 t\left(\tilde{H}_{n}^{\prime}\right)^{3}+\left(\tilde{H}_{n}^{\prime}\right)^{2}\left[4 \tilde{H}_{n}+(\alpha+2 \beta+t)^{2}+4 n(n+\alpha+\beta)-4 \beta(\alpha+\beta)\right] \\
& -2 \tilde{H}_{n}^{\prime}\left[(\alpha+2 \beta+t) \tilde{H}_{n}+2 n \beta(n+\alpha+\beta)\right]+\tilde{H}_{n}^{2}
\end{aligned}
$$

which is the Jimbo-Miwa-Okamoto $\sigma$-form of the Painleve $V$, with parameters $v_{0}=0, v_{1}=$ $-(n+a+b), v_{2}=n, v_{3}=-b$, following the convention in [33] (C.45).

\section{Painlevé V}

In this section, we obtain a second-order ordinary differential equation for $R_{n}(s)$, which is expected for $P_{V}$ since we saw that $H_{n}(s)$ satisfies the Jimbo-Miwa-Okamoto $\sigma$-form of the Painlevé V. For this purpose, we state the next lemma, a Riccati equation satisfied by $R_{n}(s)$.

Lemma 7.

$-4 s R_{n}^{\prime}(s)=4 R_{n}\left(\widetilde{r}_{n}-2 r_{n}\right)+(2 n+1+\alpha+\beta)\left(4 r_{n}+R_{n}-2 \beta\right)+2 R_{n}\left(-4 R_{n}+2 \beta+s\right)$.

Proof. First, we apply $s \frac{d}{d s}$ to (48) and make use of $\left(T_{1}\right)$ to replace $s \frac{d}{d s} \alpha_{n}$ by $\widetilde{r}_{n}-\widetilde{r}_{n+1}$. In the next step, we replace $\widetilde{r}_{n+1}$ by $\left(1-\alpha_{n}\right) \widetilde{R}_{n}-s-\widetilde{r}_{n}$ using (30). Finally, noting (48) and (31), we arrive at (80).

From (80), we see that:

$$
\widetilde{r}_{n}=\frac{-4 s R_{n}^{\prime}(s)-(2 n+1+\alpha+\beta)\left(4 r_{n}+R_{n}-2 \beta\right)}{4 R_{n}}+\frac{4 r_{n}+4 R_{n}-2 \beta-s}{2} .
$$

Substituting the above formula into (75) and (76), we find a pair of linear equations in $r_{n}(s)$ and $r_{n}^{\prime}(s)$. Solving this system, we have:

$$
\begin{aligned}
& r_{n}(s)=F\left(R_{n}, \widetilde{R}_{n}\right), \\
& r_{n}^{\prime}(s)=G\left(R_{n}, \widetilde{R}_{n}\right) .
\end{aligned}
$$


Because the expressions are unwieldy, we decided to take a different approach to obtain the Painlevé equation. In the paper of Chen and Dai [15], they studied the PollaczekJacobi-type weight, i.e.,

$$
\hat{w}(x, t)=x^{\alpha}(1-x)^{\beta} \mathrm{e}^{-\frac{t}{x}}, x \in[0,1], \alpha, \beta \geq 0, t \geq 0 .
$$

The orthogonality with the Pollaczek-Jacobi-type weight is:

$$
\int_{0}^{1} \hat{P}_{j}(x, \alpha, \beta) \hat{P}_{k}(x, \alpha, \beta) x^{\alpha}(1-x)^{\beta} \mathrm{e}^{-\frac{t}{x}} d x=\hat{h}_{j}(t, \alpha, \beta) \delta_{j k}
$$

where $\hat{P}_{j}(x, \alpha, \beta), j=0,1,2, \ldots$, are the monic polynomials of degree $j$ orthogonal with respect to the weight $\hat{w}(x, t)$. Obviously, $\hat{P}_{j}(x, \alpha, \beta)$ should also depend on $t$, while we do not write it down for simplicity.

Based on the variable substitution method for the integral, we obtained the following result.

Lemma 8. We have:

$$
\left.h_{n}(s)\right|_{s=2 t}=2^{2 n+\alpha+\beta+1} \hat{h}_{n}(t),
$$

and:

$$
\left.R_{n}(s)\right|_{s=2 t}=\frac{1}{2} \hat{R}_{n}(t),
$$

where $\hat{R}_{n}(t)$ is the quantity $R_{n}(t)$ in the paper of Chen and Dai [15].

Proof. Replacing $x$ by $\frac{1-y}{2}$, we obtain:

$$
\begin{aligned}
\hat{h}_{n}(t) & =\int_{0}^{1} \hat{P}_{n}^{2}(x) \hat{w}(x, t) d x \\
& =\int_{1}^{-1} \hat{P}_{n}^{2}\left(\frac{1-y}{2}\right)\left(\frac{1-y}{2}\right)^{\alpha}\left(\frac{1+y}{2}\right)^{\beta} \mathrm{e}^{-\frac{2 t}{1-y}} \frac{-d y}{2} \\
& =\frac{1}{2^{2 n+\alpha+\beta+1}} \int_{-1}^{1} P_{n}^{2}(y) w(y, 2 t) d y \\
& =\frac{h_{n}(2 t)}{2^{2 n+\alpha+\beta+1}} .
\end{aligned}
$$

Similarly, we obtain the relation for $R_{n}(s)$ and $\hat{R}_{n}(t)$.

Remark 5. By the variable substitution method for the integral, we obtain the relations of the auxiliary quantities between this paper and the paper of Chen and Dai [15] as follows:

$$
\begin{aligned}
& \left.\widetilde{R}_{n}(s)\right|_{s=2 t}=\hat{R}_{n}^{*}(t),\left.\quad r_{n}(s)\right|_{s=2 t}=-\hat{r}_{n}(t),\left.\widetilde{r}_{n}(s)\right|_{s=2 t}=-2 \hat{r}_{n}^{*}(t), \\
& \left.\alpha_{n}(s)\right|_{s=2 t}=1-2 \hat{\alpha}_{n}(t),\left.\quad \beta_{n}(s)\right|_{s=2 t}=4 \hat{\beta}_{n}(t) .
\end{aligned}
$$

By Theorem (7.2) in the paper of Chen and Dai [15], we have the following result :

Theorem 2. Let:

$$
S_{n}(t):=\frac{2 R_{n}(2 t)}{2 n+\alpha+\beta+1} .
$$


Then, $S_{n}(t)$ satisfies the following differential equation:

$$
\begin{aligned}
S_{n}^{\prime \prime} & =\frac{3 S_{n}-1}{2 S_{n}\left(S_{n}-1\right)}\left(S_{n}^{\prime}\right)^{2}-\frac{S_{n}^{\prime}}{t}+\frac{\left(S_{n}-1\right)^{2}}{t^{2}}\left[\frac{(2 n+1+\alpha+\beta)^{2}}{2} S_{n}-\frac{\beta^{2}}{2 S_{n}}\right] \\
& +\frac{\alpha S_{n}}{t}-\frac{S_{n}\left(S_{n}+1\right)}{2\left(S_{n}-1\right)},
\end{aligned}
$$

where is $P_{V}\left((2 n+1+\alpha+\beta)^{2} / 2,-\beta^{2} / 2, \alpha,-1 / 2\right)$ with the initial conditions:

$$
S_{n}(0)=1, \quad S_{n}^{\prime}(0)=\frac{1}{\alpha} .
$$

Proof. Equation (89) follows from the relation for $R_{n}(s)$ and $\hat{R}_{n}(t)$.

Corollary 6. Let:

$$
y_{n}(s):=\frac{2 R_{n}(s)}{2 n+\alpha+\beta+1} .
$$

Then, $y_{n}(s)$ satisfies the following differential equation:

$$
\begin{aligned}
y_{n}^{\prime \prime} & =\frac{3 y_{n}-1}{2 y_{n}\left(y_{n}-1\right)}\left(y_{n}^{\prime}\right)^{2}-\frac{y_{n}^{\prime}}{s}+\frac{\left(y_{n}-1\right)^{2}}{s^{2}}\left[\frac{(2 n+1+\alpha+\beta)^{2}}{2} y_{n}-\frac{\beta^{2}}{2 y_{n}}\right] \\
& +\frac{\alpha y_{n}}{2 s}-\frac{y_{n}\left(y_{n}+1\right)}{8\left(y_{n}-1\right)},
\end{aligned}
$$

with the initial conditions:

$$
y_{n}(0)=1, \quad y_{n}^{\prime}(0)=\frac{1}{2 \alpha}
$$

Remark 6. We made use of the ladder operator approach with the associated compatibility conditions (15), (16), and (17), since it is straightforward to express the recurrence coefficients in terms of the auxiliary variables. This allows us to investigate the sevolution of such quantities and discover the Painvelé $V$ of our problem. The Riemann-Hilbert approach of Deift et al. [34] is particularly suited for the asymptotic analysis. However, the appearance of an irregular singularity at the right endpoint requires further study.

\section{Double-Scaling Analysis}

In this section, our interest lies in a double-scaling analysis, where $s \rightarrow 0^{+}$and $n \rightarrow \infty$, such that $\tau=n^{2} s$ is finite. Recalling (41) and (63), we have:

$$
H_{n}(s)=s \frac{d}{d s} \ln \frac{D_{n}(s)}{D_{n}(0)}=(2 n+\alpha+\beta)\left(r_{n}-\widetilde{r}_{n} / 2\right)+n(\alpha+n-s / 2) .
$$

We are concerned with the behavior of the Hankel determinant, as $n$, the dimension of the Hankel matrix, tends to infinity. For this purpose, a double-scaling scheme is introduced, namely sending $n \rightarrow \infty, s \rightarrow 0^{+}$and such that $\tau:=n^{2} s$ remains fixed. Here, the main idea comes from the paper [35].

Carrying out the double scaling above and combining with Theorem 1 , we find that the infinite-dimensional Hankel determinant satisfies another $\sigma$-form of the corresponding Painlevé equation.

Theorem 3. Let:

$$
\tau:=n^{2} s,
$$


with $s \rightarrow 0^{+}$and $n \rightarrow \infty$, such that $\tau \in(0,+\infty)$. If:

$$
\mathcal{H}(\tau):=\lim _{n \rightarrow \infty} H_{n}\left(\frac{\tau}{n^{2}}\right)
$$

then $\mathcal{H}(\tau)$ satisfies,

$$
\left(\tau \mathcal{H}^{\prime \prime}\right)^{2}+4\left(\mathcal{H}^{\prime}\right)^{2}\left(\tau \mathcal{H}^{\prime}-\mathcal{H}\right)-\left(\alpha \mathcal{H}^{\prime}+\frac{1}{2}\right)^{2}=0
$$

with the initial conditions $\mathcal{H}(0)=0, \mathcal{H}^{\prime}(0)=-\frac{1}{2 \alpha}$.

Proof. Plugging $\tau=n^{2} s$ into (77), we see that the limit of $H_{n}(s)$ satisfies a $\sigma$-form Painlevé Equation (94).

Similarly, carrying out the double scaling above and combining with Corollary 6 , the scaled quantity satisfies a Painlevé III' in the next theorem.

Theorem 4. Let:

$$
y_{n}(s):=1+\frac{g_{n}(s)}{n^{2}}, \tau:=n^{2} s,
$$

with $s \rightarrow 0^{+}$and $n \rightarrow \infty$, such that $\tau \in(0,+\infty)$. If:

$$
g(\tau):=\lim _{n \rightarrow \infty} g_{n}\left(\frac{\tau}{n^{2}}\right),
$$

then $g(\tau)$ satisfies,

$$
g^{\prime \prime}(\tau)=\frac{g^{\prime 2}(\tau)}{g(\tau)}-\frac{g^{\prime}(\tau)}{\tau}+\frac{2 g^{2}(\tau)}{\tau^{2}}+\frac{\alpha}{2 \tau}-\frac{1}{4 g(\tau)},
$$

with the initial data $g(0)=0, g^{\prime}(0)=\frac{1}{2 \alpha}$.

Proof. Substituting the definition (95) into (91), we see that $g(\tau)$ satisfies $P_{I I I^{\prime}}(8,2 \alpha, 0,-1)$.

\section{Conclusions}

In this work, we studied the weight, $w(x, s):=(1-x)^{\alpha}(1+x)^{\beta} \mathrm{e}^{-\frac{s}{1-x}}, \alpha, \beta>0, \quad x \in$ $[-1,1], \quad s \geq 0$. We call this the single compression model because the weight vanishes infinitely fast at $x=1$. For the finite $n$ case, we derived a second-order nonlinear differential equation for the logarithmic derivative of the Hankel determinant. This quantity can be expressed in terms of the Jimbo-Miwa-Okamoto $\sigma$-function of a particular Painlevé V. Furthermore, we showed that the quantity $R_{n}(s)$ satisfies the four Painlevé equations. For the large $n$ case, we used double-scaling analysis and obtained similar results to the paper [35].

The main idea of this paper was to study the relationship between the Painlevé equations and the correlation coefficients, and then, we analyzed the asymptotic property of the auxiliary quantity by the double-scaling method, and finally, we obtained the asymptotic expression of the Hankel determinant.

In the future, we want to use the Riemann-Hilbert method to obtain the asymptotic expression of the orthogonal polynomials directly; thus, we can obtain the asymptotic property of the recursion coefficients of the associated orthogonal polynomials and the asymptotic expression of the Hankel determinant. 
Author Contributions: Formal analysis, P.H.; Investigation, P.H.; Project administration, Y.C.; Supervision, Y.C. All authors have read and agreed to the published version of the manuscript.

Funding: This work was supported in part by the Fundamental Research Funds for the Central Universities of China (Grant No. 2662019QD006).

Institutional Review Board Statement: Not applicable.

Informed Consent Statement: Not applicable.

Data Availability Statement: The data used to support the findings of this study are available from the corresponding author upon request.

Acknowledgments: Y. Chen would like to thank the Macau Science and Technology Development for the research grant, FDCT 079/2020/A2.

Conflicts of Interest: The authors declare no conflict of interest.

\section{References}

1. Han, P.; Chen, Y. The recurrence coefficients of a semi-classical Laguerre polynomials and the large $\mathrm{n}$ asymptotics of the associated Hankel determinant. Random Matrices Theor. Appl. 2017, 6, 1740002. [CrossRef]

2. Mehta, M.L. Random Matrices, 3rd ed.; Elsevier: New York, NY, USA, 2004.

3. Szegő, G. Orthogonal Polynomials, 4th ed.; American Mathematical Society: Providence, RI, USA, 1975.

4. Berestycki, N.; Webb, C.; Wong, M.D. Random Hermitian matrices and Gaussian multiplicative chaos. Probab. Theory Relat. Fields 2018, 172, 103-189. [CrossRef]

5. Charlier, C. Asymptotics of Hankel determinants with a one-cut regular potential and Fisher-Hartwig singularities. Int. Math. Res. Not. 2019, 24, 7515-7576. [CrossRef]

6. Charlier, C.; Gharakhloo, R. Asymptotics of Hankel determinants with a Laguerre-type or Jacobi-type potential and FisherHartwig singularities. Adv. Math. 2021, 383, 107672. [CrossRef]

7. Chen, M.; Chen, Y. Singular linear statistics of the Laguerre unitary ensemble and Painlevé III. Double scaling analysis. J. Math. Phys. 2015, 56, 063506. [CrossRef]

8. Han, P.; Chen, Y. A degenerate Gaussian weight connected with Painlevé equations and Heun equations. Random Matrices Theory Appl. 2020, 2150034. [CrossRef]

9. Lyu, S.; Chen, Y.; Fan, E. Asymptotic gap probability distributions of the Gaussian unitary ensembles and Jacobi unitary ensembles. Nucl. Phys. B 2018, 926, 639-670. [CrossRef]

10. Min, C.; Lyu, S.; Chen, Y. Painlevé III' and the Hankel determinant generated by a singularly perturbed Gaussian weight. Nucl. Phys. B 2018, 936, 169-188. [CrossRef]

11. Wu, X.; Xu, S.; Zhao, Y. Gaussian unitary ensemble with boundary spectrum singularity and $\sigma$-form of the Painlevé II equation. Stud. Appl. Math. 2018, 140, 221-251. [CrossRef]

12. Xu, S.; Dai, D.; Zhao, Y. Painlevé III asymptotics of Hankel determinants for a singularly perturbed Laguerre weight. J. Approx. Theory 2015, 192, 1-18. [CrossRef]

13. Xu, S.; Dai, D.; Zhao, Y. Hankel determinants for a singular complex weight and the first and third Painlevé transcendents. J. Approx. Theory 2016, 205, 64-92. [CrossRef]

14. Chen, Y.; Its, A. Painlevé III and a singular linear statistics in Hermitian random matrix ensembles, I. J. Approx. Theory 2010, 162, 270-297. [CrossRef]

15. Chen, Y.; Dai, D. Painlevé V and a Pollaczek-Jacobi type orthogonal polynomials. J. Approx. Theory 2010, 162, $2149-2167$. [CrossRef]

16. Min, C.; Chen, Y. Painleve V and the Hankel determinant for a singularly perturbed Jacobi weight. Nucl. Phys. B 2020, 961, 115221. [CrossRef]

17. Min, C.; Chen, Y. Differential, difference, and asymptotic relations for Pollaczek-Jacobi type orthogonal polynomials and their Hankel determinants. Stud. Appl. Math. 2021, 147, 390-416. [CrossRef]

18. Zhu, M.; Li, C.; Chen, Y. Painleve V for a Jacobi unitary ensemble with random singularities. Appl. Mathmatics Lett. 2021, 120, 107242. [CrossRef]

19. Magnus, A. Painlevé-type differential equations for the recurrence coefficients of semi-classical orthogonal polynomials. $J$. Comput. Appl. Math. 1995, 57, 215-237. [CrossRef]

20. Bonan, S.; Nevai, P. Theory orthogonal polynomials and their derivatives. I. J. Approx. Theory 1984, 40, 134-147. [CrossRef]

21. Wang, Z.; Fan, E. Critical edge behavior in the singularly perturbed Pollaczek-Jacobi type unitary ensemble. arXiv 2020, arXiv:2002.11971.

22. Texier, C.; Majumdar, S.N. Wigner time-delay distribution in chaotic cavities and freezing transition. Phys. Rev. Lett. 2013, 110, 250602. [CrossRef]

23. Brightmore, L.; Mezzadri, F.; Mo, M.Y. A matrix model with a singular weight and Painlevé III. Commun. Math. Phys. 2015, 333, 1317-1364. [CrossRef] 
24. Chen, M.; Chen, Y.; Fan, E. The Riemann-Hilbert analysis to the Pollaczek-Jacobi type orthogonal polynomials. Stud. Appl. Math. 2019, 143, 42-80. [CrossRef]

25. Barnes, E.W. The theory of the G-function. Quart. J. Math. 1900, 31, 264-314.

26. Voros, A. Spectral functions, special functions and the Selberg zeta function. Commun. Math. Phys. 1987, 110, 439-465. [CrossRef]

27. Ismail, M. Classical and Quantum Orthogonal Polynomials in One Variable. In Encyclopedia of Mathematics and Its Applications 98; Cambridge University Press: Cambridge, UK, 2005.

28. Dai, D.; Zhang, L. Painlevé VI and Hankel determinants for the generalized Jacobi weight. J. Phys. A Math. Theor. $2010,43,055207$. [CrossRef]

29. Min, C.; Chen, Y. Painlevé V, Painlevé XXXIV and the degenerate Laguerre unitary ensemble. Random Matrices Theor. Appl. 2020, 9, 2050016. [CrossRef]

30. Chihara, T.S. An Introduction to Orthogonal Polynomials; Dover: New York, NY, USA, 1978.

31. Lebedev, N.N. Special Functions and Their Applications; Dover: New York, NY, USA, 1972.

32. Chen, Y.; Ismail, M. Jacobi Polynomials from Compatibility Conditions. Proc. Am. Math. Soc. 2005, 133, 465-472. [CrossRef]

33. Jimbo, M.; Miwa, T. Monodromy preserving deformation of linear ordinary differential equations with rational coefficients. II. Physica D 1981, 2, 407-448. [CrossRef]

34. Deift, P. Orthogonal Polynomials and Random Matrices: A Riemann-Hilbert Approach; American Mathematical Society: Providence, RI, USA, 1999.

35. Chen, M.; Chen, Y.; Fan, E. Perturbed Hankel determinant, correlation functions and Painleve equations. J. Math. Phys. 2016, 57, 023501. [CrossRef] 